\title{
Machine-learning-based diagnosis of drug-naive adult patients with attention-deficit hyperactivity disorder using mismatch negativity
}

\author{
Sungkean Kim, Ji Hyun Baek², Young Joon Kwon ${ }^{3}$, Hwa Young Lee ${ }^{3}$, Jae Hyun Yoo ${ }^{4}$, Se-hoon Shim ${ }^{3 凶}$ and Ji Sun Kim (iD ${ }^{3 凶}$ \\ (c) The Author(s) 2021
}

Relatively little is investigated regarding the neurophysiology of adult attention-deficit/hyperactivity disorder (ADHD). Mismatch negativity $(\mathrm{MMN})$ is an event-related potential component representing pre-attentive auditory processing, which is closely associated with cognitive status. We investigated MMN features as biomarkers to classify drug-naive adult patients with ADHD and healthy controls (HCs). Sensor-level features (amplitude and latency) and source-level features (source activation) of MMN were investigated and compared between the electroencephalograms of 34 patients with ADHD and $45 \mathrm{HCs}$ using a passive auditory oddball paradigm. Correlations between MMN features and ADHD symptoms were analyzed. Finally, we applied machine learning to differentiate the two groups using sensor- and source-level features of MMN. Adult patients with ADHD showed significantly lower MMN amplitudes at the frontocentral electrodes and reduced MMN source activation in the frontal, temporal, and limbic lobes, which were closely associated with MMN generators and ADHD pathophysiology. Source activities were significantly correlated with ADHD symptoms. The best classification performance for adult ADHD patients and HCs showed an $81.01 \%$ accuracy, $82.35 \%$ sensitivity, and $80.00 \%$ specificity based on MMN source activity features. Our results suggest that abnormal MMN reflects the adult ADHD patients' pathophysiological characteristics and might serve clinically as a neuromarker of adult ADHD.

Translational Psychiatry (2021)11:484; https://doi.org/10.1038/s41398-021-01604-3

\section{INTRODUCTION}

Attention-deficit/hyperactivity disorder (ADHD) has been defined as a neurodevelopmental disorder with symptoms of distracted attention, hyperactivity, and impulsivity [1]. Although ADHD is known to be a disease with childhood-onset, $\sim 15 \%$ of children with ADHD maintain their symptomatology, meeting full diagnostic criteria for adults [2]. Detection of adult patients with ADHD is crucial to providing proper management because they still display marked difficulties in maintaining selective attention to relevant information and increased distractibility towards irrelevant stimuli [3, 4]. However, ADHD in adulthood is often misdiagnosed as anxiety or mood symptoms in a clinical setting [5]. Despite the existence of adulthood ADHD and its clinical importance, relatively little is known about the neurobiological background of adult ADHD [6].

Event-related potentials (ERPs) have been widely utilized to study the attentional processes in childhood ADHD [7]. In particular, mismatch negativity (MMN), an ERP component, represents pre-attentive auditory processing that is closely correlated with cognitive status in the absence of behavioral responses as well as motivation [8-10]. Given that patients with ADHD suffer from proper distribution of attention, such as focusing and maintaining their attention on the tasks, MMN has been considered a good neuromarker for evaluating the neurophysiological mechanisms of ADHD. Moreover, MMN reflects glutamatergic function [11], and the critical pathology for MMN reduction might be associated with the $\mathrm{N}$-methyl-D-aspartate (NMDA) receptor system dysfunction [12]. NMDA receptors have been well studied for their crucial role in cognitive function, such as learning and memory $[13,14]$. In addition, recent reports also suggest the dysfunction of NMDA receptors in the pathophysiology of ADHD [15]. In this regard, MMN might be a promising biomarker of ADHD.

Several studies have assessed MMN changes and their correlates with attentional problems in children with ADHD [16-20]. A recent meta-analysis revealed a reduced MMN amplitude in children with ADHD compared to healthy controls (HCs) [7]. However, to date, no study has investigated MMN changes in adult ADHD. Regarding maturational processes of brain activity with aging [21], the neurobiological basis of adult ADHD might be different from that of childhood ADHD. In addition, even though the symptoms of adult ADHD are similar to those of childhood ADHD, symptom severity, especially hyperactivity, may decrease over time [22]. This suggests that adults with ADHD may display different MMN responses from those of childhood ADHD.

Recently, an increasing number of researchers have attempted to differentiate patients with ADHD from HCs on the basis of

\footnotetext{
${ }^{1}$ Department of Human-Computer Interaction, Hanyang University, Ansan, Republic of Korea. ${ }^{2}$ Department of Psychiatry, Samsung Medical Center, Sungkyunkwan University School of Medicine, Seoul, Republic of Korea. ${ }^{3}$ Department of Psychiatry, Soonchunhyang University Cheonan Hospital, Cheonan, Republic of Korea. ${ }^{4}$ Department of Psychiatry, Seoul St. Mary's Hospital, College of Medicine, The Catholic University of Korea, Seoul, Republic of Korea. ${ }^{凶}$ email: shshim2k@daum.net; ideal91@hanmail.net
}

Received: 7 December 2020 Revised: 23 August 2021 Accepted: 1 September 2021

Published online: 18 September 2021 
machine learning methods with EEG biomarkers. However, few studies have investigated the classification of adult patients with ADHD and HCs. They have reported accuracies ranging from hardly above chance level (50\%) to beyond $90 \%$. For example, Mueller et al. [23, 24] classified adult ADHD patients and HCs using Go-NoGo ERPs and achieved accuracies above $90 \%$. Tenev et al. [25] measured resting-state conditions and neuropsychological tasks, obtaining an accuracy of $82 \%$. Studies of resting-state band power reported $68 \%$ accuracy [21] and 76\% accuracy [26]. In addition, Kiiski et al. [6] demonstrated that resting-state connectivity features did not reliably classify ADHD patients and $\mathrm{HCs}$.

Detection of electrophysiological markers to differentiate adult ADHD patients could support clinicians to provide appropriate diagnosis and treatment for patients suffering from difficulties in directing and maintaining attention in their lives. However, given that there have been limited studies of adult ADHD, further studies are warranted to identify biomarkers for adult ADHD using machine learning techniques. Moreover, research differentiating between adult patients with ADHD and HCs using MMN features has not yet been undertaken.

The aim of this study was to investigate changes in MMN features in adult patients with ADHD compared to HCs at both the sensor (amplitude and latency) and source levels (source activation). In addition, we explored the relationships between MMN features and ADHD symptom scores. Finally, we examined the possibility of $\mathrm{MMN}$ features serving as biomarkers by differentiating between ADHD patients and HCs using machine learning techniques. We hypothesized that MMN activities at both the sensor and source levels would be attenuated in adult ADHD patients compared to $\mathrm{HCs}$, reflecting the pathophysiology of ADHD. We also hypothesized that these MMN characteristics could differentiate between ADHD patients and HCs with acceptable classification performances. To the best of our knowledge, this study is the first to examine the differences in MMN characteristics between adult patients with ADHD and HCs and to classify them via machine learning measures.

\section{MATERIALS AND METHODS \\ Participants}

A total of 79 subjects aged $18-45$ years participated in this study. Subjects included patients with ADHD ( $n=34$, male: 28 , female: 6 , mean age: $24.76 \pm 7.02$ years; range: $19-45$ years) and healthy controls ( $n=45$, male: 36, female: 9, mean age: $25.51 \pm 5.48$ years; range: $18-37$ years). Participants with ADHD were enrolled from the Department of Psychiatry at Soonchunhyang University Cheonan Hospital, Korea. All psychiatric evaluations were conducted by a board-certified psychiatrist specializing in adult ADHD using the full criteria for ADHD in accordance with the DSM-V. Patients who had mental retardation or alcohol abuse, undergone electroconvulsive therapy, or suffered head injury were not included. All patients with ADHD were drug-naïve. Forty-five non-smoking HCs were enrolled by the local community via newspapers and posters. We excluded participants with any axis I or II comorbid psychiatric diagnosis or any history of neurological diseases from the initial screening interviews. Participants had normal hearing ability confirmed by the $512 \mathrm{~Hz}$ tuning fork test [27], and all were identified as right-handed. The Institutional Review Board and Ethics Committee of Soonchunhyang University Cheonan Hospital approved the study and all experimental protocols (IRB number: 2019-05-004). The study was conducted according to the approved guidelines. Informed consent was acquired from all study participants.

\section{Psychological measures}

All participants were assessed for ADHD symptoms using the Korean version of the Adult ADHD self-report scales (ASRS) [28]. The ASRS is a widely used self-reporting scale with 18 items scored on a 5-point Likert scale to screen for ADHD in the general population [29]. It evaluates ADHD symptoms based on the DSM-IV criteria for ADHD over the past six months. Inattention (ASRS inattention score, ASRS-I) and hyperactivity scores (ASRS hyperactivity score, ASRS-H) were calculated separately. The Korean version of the ASRS shows good sensitivity and specificity [28].

\section{Data acquisition and analysis}

EEG data were recorded by a NeuroScan SynAmps2 amplifier (Compumedics USA, Charlotte, NC, USA) with $62 \mathrm{Ag}$-AgCl channels mounted on a Quik Cap, using an extended 10-20 placement scheme. The ground channel was placed on the forehead, and the physically linked reference channel was attached to both mastoids. Vertical electrooculogram (EOG) channels were located above and below the left eye. Horizontal EOG channels were placed at the outer canthus of each eye. The impedance was maintained below $5 \mathrm{k} \Omega$. The EEG data were obtained with a band-pass filter with cutoff frequencies ranging from 0.1 to $100 \mathrm{~Hz}$ at a $1000 \mathrm{~Hz}$ sampling rate.

The acquired EEG data were preprocessed by CURRY 8 (Compumedics USA, Charlotte, NC, USA) and MATLAB R2018b (MathWorks, Natick, MA, USA). Gross artifacts were rejected from visual inspection of an experienced person without any prior knowledge concerning the origin of the data. Artifacts related to eye movements or eye blinks were corrected using a covariance- and regression-based mathematical procedure implemented in the preprocessing software [30]. The data were filtered using a $1-30 \mathrm{~Hz}$ band-pass filter. Then, the data were epoched from $100 \mathrm{~ms}$ pre-stimulus to $600 \mathrm{~ms}$ post-stimulus. For baseline correction, the epochs were deducted from the mean value of the prestimulus interval. If there were any remaining epochs containing significant physiological artifacts (amplitude exceeding $\pm 75 \mu \mathrm{V}$ ) in any of the 62 channel sites, they were rejected from further analyses. For the ERP analysis, only artifact-free epochs were averaged along trials and participants.

EEG recordings and stimulus presentation onset were synchronized by E-prime (Psychology Software Tools, Pittsburgh, PA, USA). The auditory stimuli composed of sounds at $1000 \mathrm{~Hz}$ and $85 \mathrm{~dB}$ SPL. The participants were instructed to focus their attention on a picture book called "Where's Wally?" without paying attention to the auditory stimuli. To obtain the MMN wave, the ERP wave derived from standard stimuli was subtracted by the ERP wave from deviant stimuli for each participant.

Standard stimuli lasting $50 \mathrm{~ms}$ were presented, randomly interspersed with deviant stimuli lasting $100 \mathrm{~ms}(90 \%$ and $10 \%$ probabilities, respectively). There were a total of 750 auditory stimuli with a $500 \mathrm{~ms}$ inter-stimulus interval. These stimuli were presented by MDR-D777 headphones (Sony, Tokyo, Japan). The experiment took about $10 \mathrm{~min}$ to complete.

MMN amplitude was measured as the peak voltage between 130 and $280 \mathrm{~ms}$ at nine channels ( $\mathrm{F} 3, \mathrm{Fz}, \mathrm{F} 4, \mathrm{FC} 3, \mathrm{FCZ}, \mathrm{FC} 4, \mathrm{C} 3, \mathrm{CZ}$, and C4) according to previous studies revealing that the frontocentral electrodes show larger MMN amplitudes [31-33]. The time range for MMN peak amplitudes was on the basis of the grand-averaged waveforms at FCz channel. The number of epochs for standard and deviant stimuli in the analysis did not significantly differ between patients with ADHD and HCs (standard stimuli: $598.59 \pm 66.12$ vs. $609.18 \pm 48.56, p=0.414$; deviant stimuli: $66.91 \pm 7.46$ vs. $67.71 \pm 5.14, p=0.575)$.

\section{Source imaging}

In order to estimate the cortical distribution of the standardized source current density for MMN activity, standardized low-resolution brain electromagnetic tomography (sLORETA) was employed. sLORETA has been widely utilized as a representative source-imaging method to solve the EEG inverse problem [34]. It assumes that the source activity of a voxel is similar to that of the neighboring voxels when computing a particular solution, and applies a proper standardization for the current density. The lead field matrix was calculated using a realistic head model which was segmented according to the Montreal Neurological Institute (MNI) 152 standard template, wherein the three-dimensional solution space was confined to only the cortical gray matter and hippocampus [35]. The three-dimensional solution space consisted of 6239 voxels with a $5-\mathrm{mm}$ resolution. Anatomical labels including the Brodmann areas were provided with a proper transformation from the MNI to Talairach space [36].

The MMN source image was analyzed between 130 and $280 \mathrm{~ms}$ after stimulus onset. The comparison of sLORETA images between adult patients with ADHD and HCs for MMN was conducted by a statistical non-parametric mapping method (SnPM) carried out in the sLORETA software. The estimated voxel activation was averaged across the calculated time frame and tested with a voxel-by-voxel independent $t$-test for the 6239 voxels, followed by a randomization test $(n=5000)$ for correcting multiple comparisons. 


\section{Statistical analysis}

Differences of age, education years, and psychological characteristics (ASRS) between adult patients with ADHD and HCs were compared using independent $t$-tests. A chi-squared test was used to analyze betweengroup difference in sex ratio. The significance level was $p<0.05$ (twotailed). A multivariate analysis of variance (MANOVA) was performed to evaluate differences in MMN amplitudes and latencies at frontocentral electrodes and MMN source activities between the two groups, with education years as a covariate. An adjusted $p$-value of $0.05 / 52=0.000962$ (18 features from sensor level and 34 features from source level) by Bonferroni corrections were used to control for multiple comparisons. Effect sizes were represented as partial eta squared $\left(\eta^{2}\right)$. A partial Pearson's correlation analysis was conducted between MMN features from sensor and source levels and ADHD symptom scores, with years of education controlled for. After the correlation analysis, an adjusted $p$-value of $0.05 /$ $\left(3^{*} 27\right)=0.000617$ (27 significant MMN features with three ASRS scores) by Bonferroni corrections were applied. Statistical analyses were carried out using SPSS 21 (SPSS, Inc., Chicago, IL, USA).

\section{Feature selection and classification}

We discriminated between adult ADHD patients and HCs using the MMN sensor and source activities to check their potential usability as biomarkers. To find optimal features for discriminating the two groups, both MMN features from sensor and source levels were used as follows (Table 1): sensor-level (18 features), each of the nine frontocentral $M M N$ amplitudes and latencies (F3, Fz, F4, FC3, FCz, FC4, C3, Cz, and C4); source-level (34 features), $\mathrm{MMN}$ source activities in 34 brain regions (frontal areas: superior frontal gyrus, middle frontal gyrus, medial frontal gyrus, inferior frontal gyrus, orbital gyrus, subcallosal gyrus, rectal gyrus; temporal areas: superior temporal gyrus, middle temporal gyrus, inferior temporal gyrus, transverse temporal gyrus; limbic areas; anterior cingulate cortex, insula, extra nuclear, parahippocampla gyrus, uncus, cingulate gyrus). We selected these brain regions on the basis of the former neuroimaging and ERP source localization studies for MMN generator [37-44], and the results of MMN source activities showing significant differences between the two groups in our data.

Table 1. All MMN features including sensor and source levels for classification between adult ADHD patients and healthy controls.

\section{Classification features}

\begin{tabular}{l} 
Sensor level (18 features) \\
MMN peak amplitude \\
FC3 peak amplitude \\
FCz peak amplitude \\
FC4 peak amplitude \\
F3 peak amplitude \\
Fz peak amplitude \\
F4 peak amplitude \\
C3 peak amplitude \\
Cz peak amplitude \\
C4 peak amplitude \\
MMN peak latency \\
\hline FC3 peak amplitude \\
FCz peak latency \\
FC4 peak latency \\
\hline F3 peak latency \\
\hline Fz peak latency \\
\hline F4 peak latency \\
\hline C3 peak latency \\
Cz peak latency \\
C4 peak latency \\
\hline
\end{tabular}

Source level (34 features)

MMN source activity

Frontal lobe

Bilateral superior frontal gyrus

Bilateral middle frontal gyrus

Bilateral medial frontal gyrus

Bilateral inferior frontal gyrus

Bilateral orbital gyrus

Bilateral subcallosal gyrus

Bilateral rectal gyrus

Temporal lobe

Bilateral superior temporal gyrus

Bilateral middle temporal gyrus

Bilateral inferior temporal gyrus

Bilateral transverse temporal gyrus

Limbic lobe

Bilateral anterior cingulate cortex

Bilateral insula

Bilateral extra nuclear

Bilateral parahippocampal gyrus

Bilateral uncus

Bilateral cingulate gyrus

$A D H D$ attention-deficit/hyperactivity disorder, $M M N$ mismatch negativity.
Classification was performed using a linear support vector machine classifier with the cost set as 1 [45-47] and the classification accuracy was evaluated using a leave-one-out cross-validation method for each feature set. Linear support vector machine has been widely applied in multivariate pattern analysis with its high accuracy, generalization, and interpretability [48-50]. Many studies have used the leave-one-out cross-validation method claiming that it is more appropriate for small data since more data can be trained for a classification model and that it imitates clinical setting where clinicians can learn from large data and apply the findings to new each case [51-53]. In order to decrease the computational cost and prevent potential overfitting by the large number of features, the Fisher score was used for feature selection $[54,55]$. For each cross-validation, the Fisher score was used to select the best feature subset for the current training dataset. A higher Fisher score for each feature represents better separability between the two groups. Different numbers of features with higher Fisher scores ranging from 1 to 20 were respectively examined for classification between the two groups [56]. Finally, the classification performances including accuracy, sensitivity, and specificity were averaged in the leave-one-out cross-validation.

In addition, 1000 times permutation test (group label permutation) were performed to assess the statistical significance of our classification accuracy $[47,57,58]$. We labeled each participant to two groups randomly, trained and tested the support vector machine classifier with this random labeling, and calculated the accuracy from the classification model. A MATLAB toolbox, Pattern Recognition Tools 5 (http://37steps.com) for the machine learning analyses.

\section{RESULTS}

\section{Demographic and psychological characteristics}

Table 2 presents the demographic and psychological characteristic comparison between the patients with ADHD and HCs. There was a significant difference in education years. HCs showed significantly higher education years than patients with ADHD $(p<$ 0.001). In addition, there were significant differences in ASRS scores between the two groups, such that the patients with ADHD showed significantly higher ASRS $(p<0.001)$ and its subscales, including inattention $(p<0.001)$ and hyperactivity $(p<0.001)$.

\section{Mismatch negativity}

The patients with ADHD showed significantly reduced MMN peak amplitudes compared to $\mathrm{HCs}$ at the FCz $(p<0.001)$, FC4 $(p<$ $0.001), C 3(p<0.001), C z(p<0.001)$, and C4 $(p<0.001)$ electrodes. However, there was no significant difference in MMN latency between the two groups. Figure 1 shows the grand-average waveforms and topographical distributions for MMN in each group.

\section{Source analysis}

Adult patients with ADHD revealed significantly decreased MMN source activities compared to $\mathrm{HCs}$ in the right middle frontal gyrus, right medial frontal gyrus, bilateral anterior cingulate cortex, bilateral inferior frontal gyrus, right superior temporal gyrus, right inferior temporal gyrus, bilateral insula, bilateral extra nuclear, bilateral orbital gyrus, bilateral parahippocampal gyrus, bilateral

Table 2. Demographic characteristics of study participants.

\begin{tabular}{|llll|}
\hline & ADHD $(\boldsymbol{N}=\mathbf{3 4})$ & HC $(\boldsymbol{N}=\mathbf{4 5})$ & $\boldsymbol{P}$ \\
\hline Age (years) & $24.76 \pm 7.02$ & $25.51 \pm 5.48$ & 0.597 \\
\hline Sex & & & 0.792 \\
\hline Male & $28(82.4)$ & $36(80.0)$ & \\
\hline Female & $6(17.6)$ & $9(20.0)$ & \\
\hline Education (years) & $12.82 \pm 1.40$ & $14.36 \pm 2.31$ & $<0.001$ \\
\hline $\begin{array}{l}\text { Adult ADHD Self-Report } \\
\text { Scale (ASRS) }\end{array}$ & $42.12 \pm 11.13$ & $10.60 \pm 8.98$ & $<0.001$ \\
\hline Inattention & $24.21 \pm 5.84$ & $7.27 \pm 5.61$ & $<0.001$ \\
\hline Hyperactivity & $17.91 \pm 6.15$ & $3.33 \pm 4.09$ & $<0.001$ \\
\hline
\end{tabular}

$A D H D$ attention-deficit/hyperactivity disorder, $H C$ healthy control. 
(A)

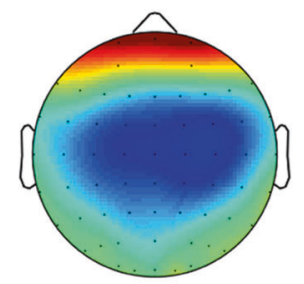

ADHD

(B)

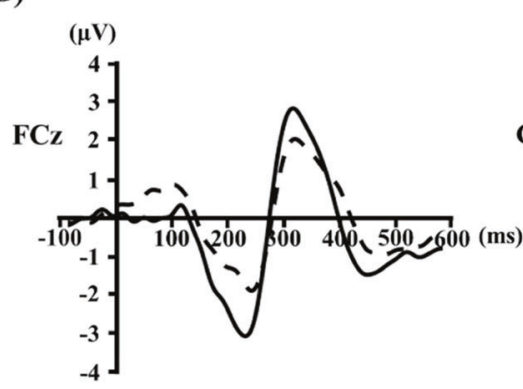

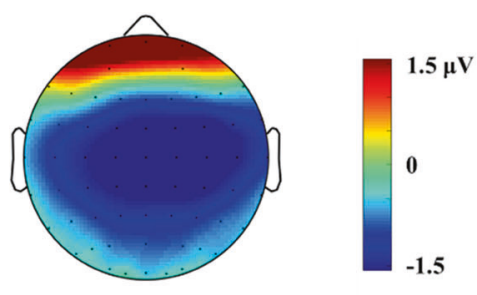

Healthy controls
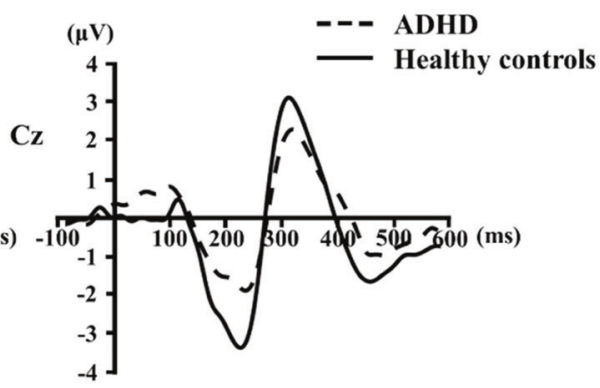

Fig. 1 Topographical distributions and grand-average waveforms for mismatch negativity (MMN) in adult patients with ADHD and healthy controls. (A) Topographic maps of MMN, and (B) Grand average of MMN waveforms at FCz and Cz electrodes.

\begin{tabular}{|c|c|c|c|c|}
\hline Region of Interest & ADHD $(N=34)$ & $\mathrm{HC}(N=45)$ & Effect size $\left(\eta^{2}\right)$ & $P$ \\
\hline \multicolumn{5}{|l|}{ Left } \\
\hline Anterior cingulate cortex & $2.74 \pm 1.96$ & $6.36 \pm 3.54$ & 0.227 & $<0.001$ \\
\hline Inferior frontal gyrus & $2.79 \pm 1.62$ & $5.46 \pm 2.87$ & 0.191 & $<0.001$ \\
\hline Rectal gyrus & $4.46 \pm 3.59$ & $10.53 \pm 6.17$ & 0.216 & $<0.001$ \\
\hline Orbital gyrus & $5.84 \pm 4.65$ & $12.98 \pm 7.99$ & 0.181 & $<0.001$ \\
\hline \multicolumn{5}{|l|}{ Right } \\
\hline Anterior cingulate cortex & $2.72 \pm 1.92$ & $6.16 \pm 3.46$ & 0.208 & $<0.001$ \\
\hline Inferior frontal gyrus & $2.64 \pm 1.78$ & $5.15 \pm 2.23$ & 0.256 & $<0.001$ \\
\hline Uncus & $2.13 \pm 1.69$ & $5.19 \pm 2.99$ & 0.248 & $<0.001$ \\
\hline Superior temporal gyrus & $1.74 \pm 1.10$ & $3.19 \pm 1.43$ & 0.237 & $<0.001$ \\
\hline
\end{tabular}

$A D H D$ attention-deficit/hyperactivity disorder, $H C$ healthy control, $M M N$ mismatch negativity.

rectal gyrus, bilateral subcallosal gyrus, and bilateral uncus $(p<$ 0.001; Table 3, Fig. 2).

\section{Correlation between MMN characteristics and ADHD symptoms}

Correlation analysis detected significant correlations between the MMN source activities and ADHD symptoms in the study population: (1) ASRS: left anterior cingulate cortex $(r=-0.427$, $p<0.001)$, right anterior cingulate cortex $(r=-0.435, p<0.001)$, right inferior frontal gyrus $(r=-0.428, p<0.001)$, right superior temporal gyrus $(r=-0.385, p<0.001)$, right extra nuclear $(r=$ $-0.413, p<0.001)$, left orbital gyrus $(r=-0.401, p<0.001)$, right orbital gyrus $(r=-0.428, p<0.001)$, left rectal gyrus $(r=-0.418$, $p<0.001)$, right rectal gyrus $(r=-0.440, p<0.001)$, left subcallosal gyrus $(r=-0.408, p<0.001)$, right subcallosal gyrus $(r=-0.446, p$
$<0.001)$, right uncus $(r=-0.401, p<0.001)$; (2) inattention: left anterior cingulate cortex $(r=-0.417, p<0.001)$, right anterior cingulate cortex $(r=-0.435, p<0.001)$, right inferior frontal gyrus $(r=-0.424, p<0.001)$, right extra nuclear $(r=-0.394, p<0.001)$, left orbital gyrus $(r=-0.396, p<0.001)$, right orbital gyrus $(r=$ $-0.434, p<0.001)$, left rectal gyrus $(r=-0.412, p<0.001)$, right rectal gyrus $(r=-0.440, p<0.001)$, left subcallosal gyrus $(r=$ $-0.393, p<0.001)$, right subcallosal gyrus $(r=-0.430, p<0.001)$, right uncus ( $r=-0.388, p<0.001)$; (3) hyperactivity: left anterior cingulate cortex $(r=-0.413, p<0.001)$, right anterior cingulate cortex $(r=-0.409, p<0.001)$, right inferior frontal gyrus $(r=$ $-0.407, p<0.001)$, right extra nuclear $(r=-0.410, p<0.001)$, left orbital gyrus $(r=-0.384, p<0.001)$, right orbital gyrus $(r=$ $-0.396, p<0.001)$, left rectal gyrus $(r=-0.401, p<0.001)$, right rectal gyrus $(r=-0.413, p<0.001)$, left subcallosal gyrus $(r=$ 
(A) Anterior cingulate cortex

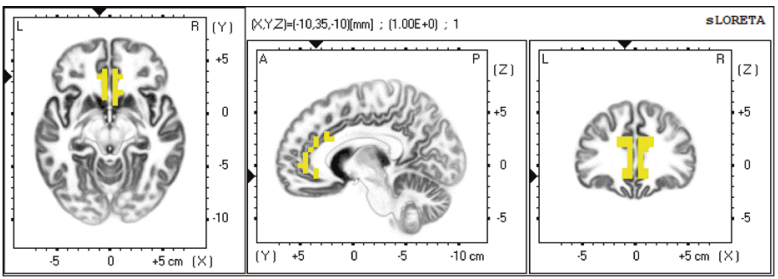

(C) Rectal gyrus

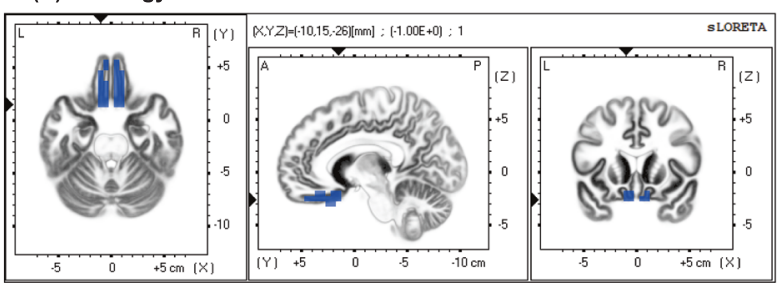

(E) Extra nuclear

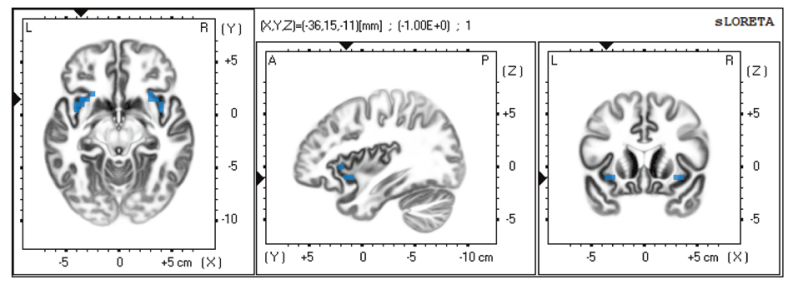

(G) Uncus

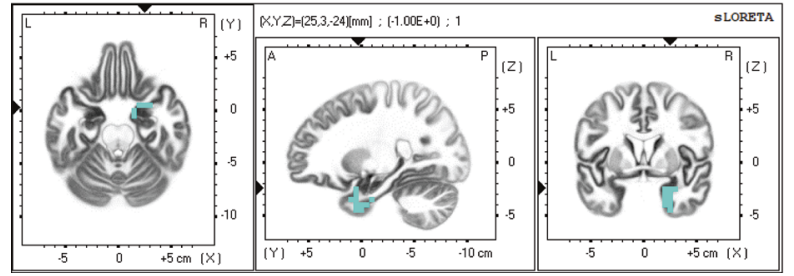

(B) Inferior frontal gyrus

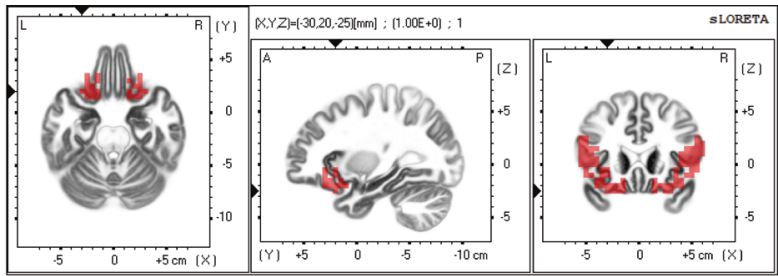

(D) Subcallosal gyrus

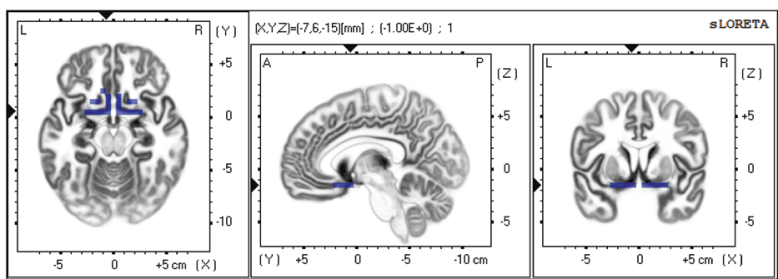

(F) Orbital gyrus

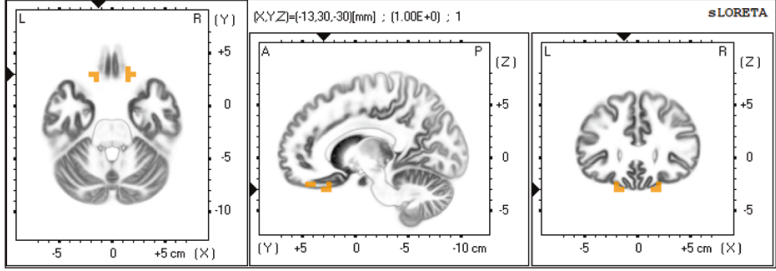

(H) Superior temporal gyrus

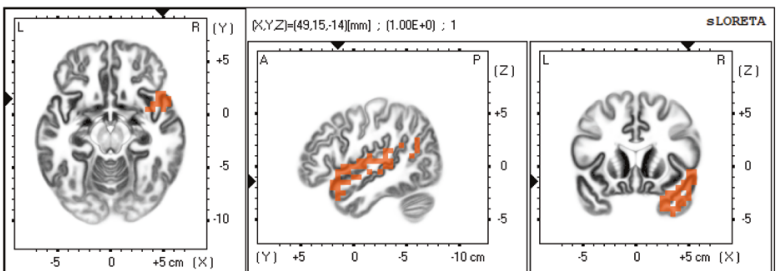

Fig. 2 Brain regions showing the best classification performances from the source-level feature set between adult ADHD patients and healthy controls. (A) Anterial cingulate cortex, (B) Inferior frontal gyrus, (C) Rectal gyrus, (D) Subcallosal gyrus, (E) Extra nuclear, (F) Orbital gyrus, (G) Uncus, and (H) Superior temporal gyrus.

$-0.401, p<0.001)$, right subcallosal gyrus $(r=-0.438, p<0.001)$ right uncus $(r=-0.393, p<0.001)$ (Fig. 3). There was no significant correlation between MMN sensor-level features and ADHD symptom scores.

\section{Classification results}

Table 4 presents the best classification performances between adult ADHD patients and HCs for three different feature sets (sensor-level, source-level, and both sensor and source levels). For the sensor-level feature set, the best classification accuracy was $78.48 \%$ with three features: MMN amplitudes at $\mathrm{FCz}, \mathrm{FC} 4$, and $\mathrm{Cz}$ electrodes. In terms of source-level feature set, the best classification accuracy was $81.01 \%$ with 14 features, MMN source activities in the bilateral anterior cingulate cortex, bilateral inferior frontal gyrus, bilateral rectal gyrus, bilateral subcallosal gyrus, bilateral extra nuclear, bilateral orbital gyrus, right uncus, and right superior temporal gyrus. For both sensor and source levels, the best classification accuracy was $81.01 \%$ with 16 features, MMN amplitude at FC4 and MMN source activities in the bilateral anterior cingulate cortex, bilateral inferior frontal gyrus, bilateral rectal gyrus, bilateral subcallosal gyrus, bilateral extra nuclear, bilateral orbital gyrus, bilateral uncus, and right superior temporal gyrus.

The permutation test results demonstrated that our classification accuracies were significant. The classification accuracies from the original data were higher than all the 1000 classification accuracies from the permutation test for the three different feature sets (mean \pm 2 standard deviation; sensor-level: $54.92 \pm$ $9.60 \%$, source-level: $52.47 \pm 13.96 \%$, both sensor and source levels: $52.03 \pm 15.21 \%)$.

\section{DISCUSSION}

In this study, we investigated MMN abnormalities, indicative of pre-attentive central processing of auditory change detection, in drug-naive adult patients with ADHD and differentiated ADHD patients and HCs based on machine learning methods. Our major findings are as follows. First, the patients with ADHD showed significantly reduced $M M N$ amplitudes at the frontocentral electrodes compared to HCs. Second, the patients exhibited significantly decreased MMN source activities in the frontal, temporal, and limbic regions. Third, MMN source activities were negatively correlated with ADHD symptom scores in the study population. Fourth, the best classification performance for adult ADHD patients and HCs was an $81.01 \%$ accuracy, an $82.35 \%$ sensitivity, and an $80.00 \%$ specificity from the MMN source activity features.

The MMN amplitudes were attenuated in adult patients with ADHD compared to HCs at the frontocentral electrodes. Although there has been no previous study exploring MMN in adult patients with ADHD, our results are comparable to those of previous studies showing decreased MMN amplitudes in children and 

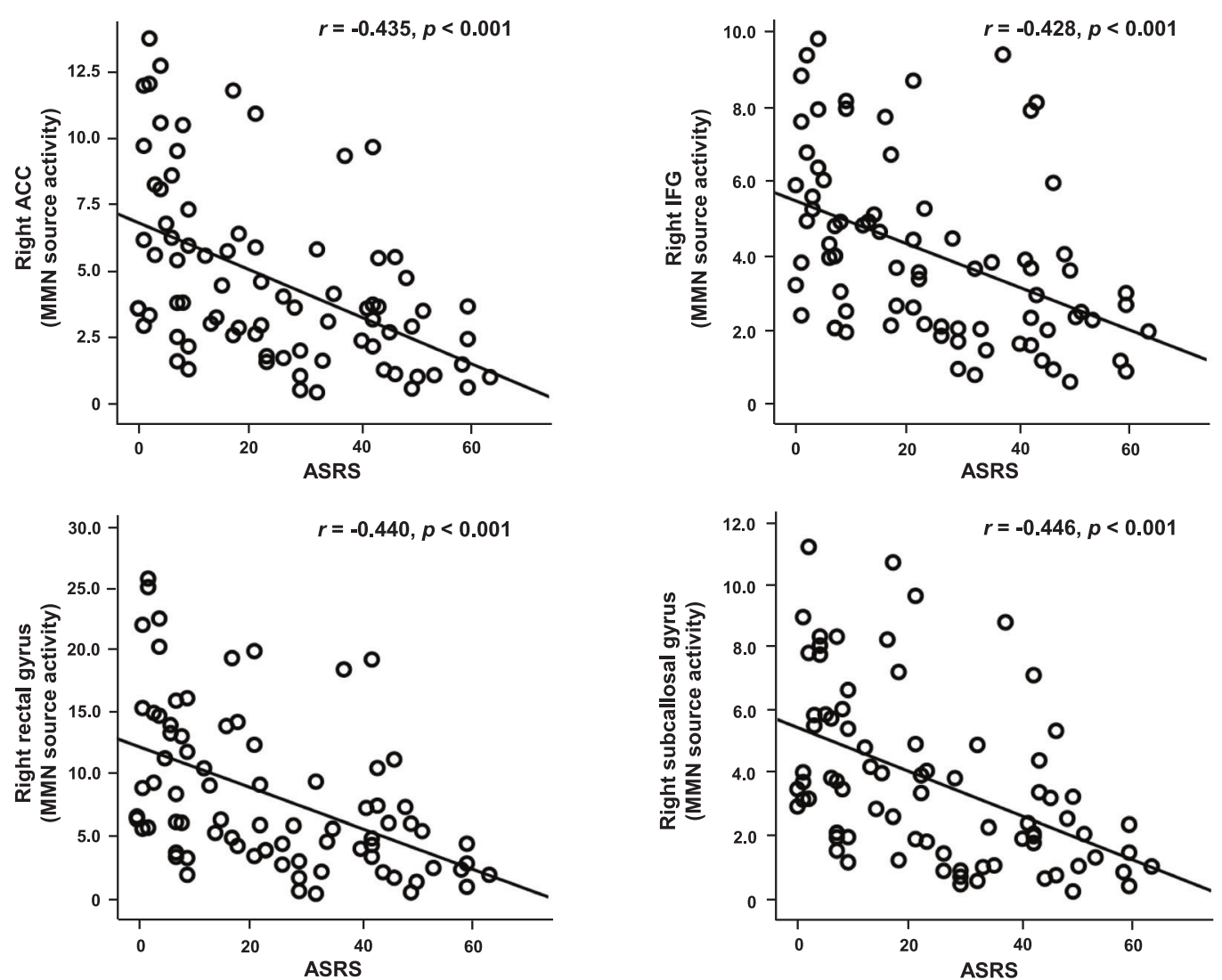

Anterior cingulate cortex (ACC)

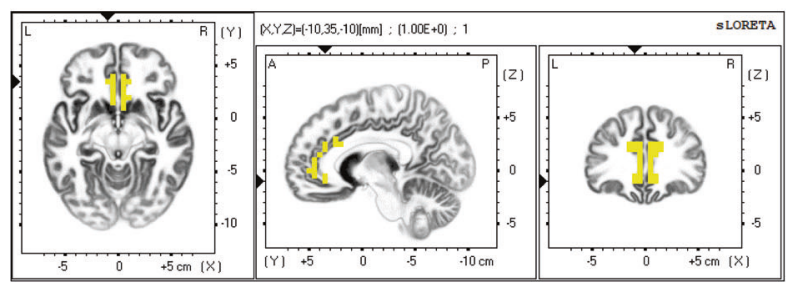

Inferior frontal gyrus (IFG)
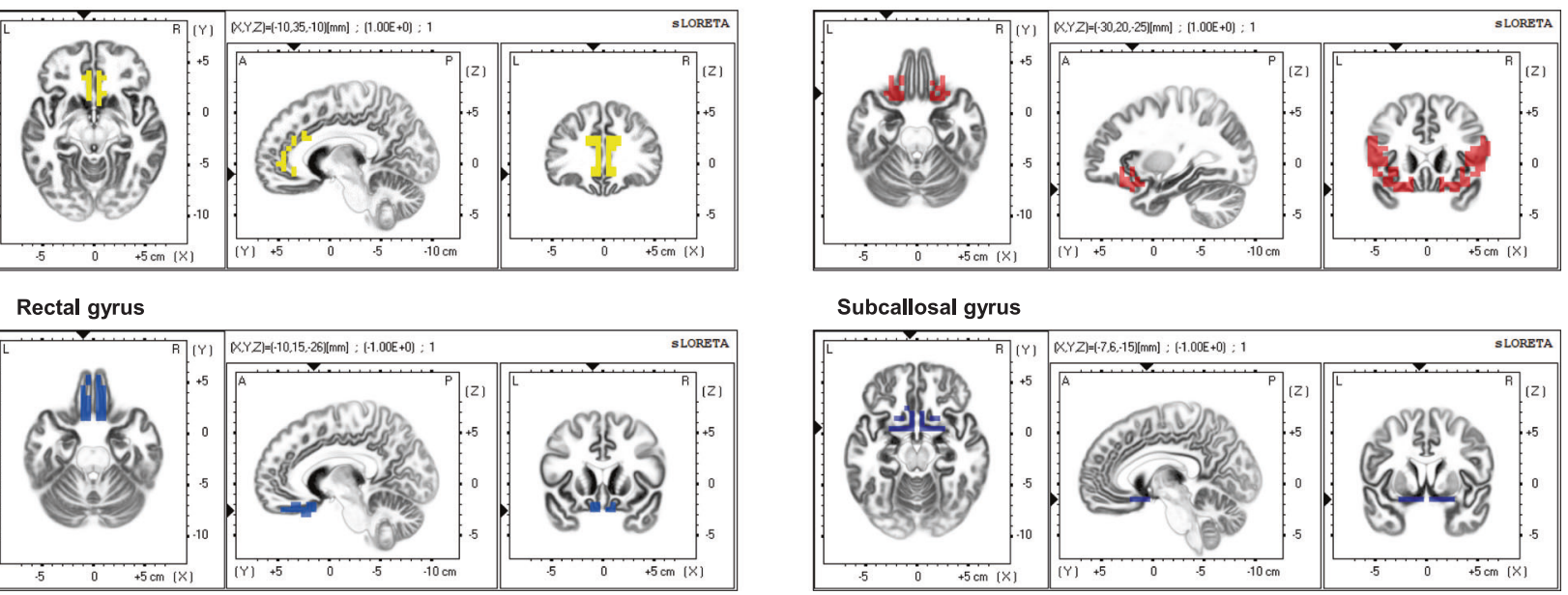

Fig. 3 Correlation between mismatch negativity (MMN) characteristics and ADHD symptoms. Correlations between MMN source activities in anterior cingulate cortex, inferior frontal gyrus, rectal gyrus, and subcallosal gyrus and ADHD symptom scores in all participants.

Table 4. The best classification performances (\%) of adult ADHD patients and healthy controls for three different feature sets (sensorlevel, source-level, and both sensor- and source-levels).

\begin{tabular}{|llll|}
\hline \multicolumn{3}{c}{ ADHD vs. HC } \\
& Accuracy & Sensitivity & Specificity \\
\hline Sensor level & 78.48 & 70.59 & 84.44 \\
\hline Source level & 81.01 & 82.35 & 80.00 \\
\hline Sensor level + source level & 81.01 & 79.41 & 82.22 \\
\hline
\end{tabular}

$A D H D$ attention-deficit/hyperactivity disorder, $H C$ healthy control. adolescent patients with ADHD [7, 17, 59]. Given the notion that MMN reflects pre-attentive detection related to cognitive status $[9,10]$, the attenuated $\mathrm{MMN}$ amplitudes suggest that adult patients with $A D H D$ also suffer from dysfunctional pre-attentive processes. Moreover, MMN has been thought to reflect information processing more accurately than the P300 ERP component, and has been closely related to attention deficit [59]. Although the $M M N$ is not subject to conscious control, the changes in MMN in adult patients with ADHD might reflect the dysfunctional attentional process of $\operatorname{ADHD}[60,61]$. Impairments in deviance detection might cause significant dysfunctions in higher-order cognitive functioning, affecting the inattentive and hyperactive symptoms of patients with ADHD. 
In this study, adult patients with ADHD showed significantly reduced MMN source activities compared to HCs in the frontal lobe (right middle frontal gyrus, right medial frontal gyrus, bilateral inferior frontal gyrus, bilateral orbital gyrus, bilateral subcallosal gyrus, bilateral rectal gyrus), temporal lobe (right superior temporal gyrus, right inferior temporal gyrus), and limbic lobe (bilateral anterior cingulate cortex, bilateral insula, bilateral extra nuclear, bilateral parahippocampal gyrus, bilateral uncus). In addition, reduced source activities in the bilateral anterior cingulate cortex, right inferior frontal gyrus, right superior temporal gyrus, right extra nuclear, bilateral orbital gyrus, bilateral rectal gyrus, bilateral subcallosal gyrus, and right uncus were negatively correlated with the ADHD symptom scales in the study population.

In general, auditory MMN is produced in the primary auditory cortex and adjacent areas of the superior temporal lobe [42, 62]. The frontal areas, including the anterior cingulate cortex and middle and inferior frontal gyrus, are also considered MMN generators [39, 42, 63-65]. Temporal generators are related to auditory feature analysis and deviance detection, and frontal generators with involuntary switching of attention toward changes in the auditory environment $[38,66]$. In particular, frontal generators have been associated with a cognitive role or comparator-based mechanism of MMN [67-69].

Regions in which we found dysfunctional MMN source activities and significant symptomatic correlations were areas of focus in several previous ADHD studies. According to previous neuroimaging studies, the development of ADHD was closely associated with impaired frontal lobe function [70, 71]. Altered neurochemistry in the anterior cingulate cortex is thought to be an important cause of behavioral symptoms of ADHD [72]. Structural abnormalities such as smaller volume and reduced cortical thickness in the anterior cingulate cortex have been observed in both children and adult patients with ADHD than in HCs [73-77]. In a variety of cognitive tasks, patients with ADHD have shown abnormal performance that might be specifically linked to structural and/or functional anomalies in the anterior cingulate cortex [78, 79].

In addition, several studies have highlighted that the inferior frontal gyrus is crucial for inhibiting behavioral responses related to impulsivity and hyperactivity [80, 81]. Opitz et al. [82] suggested that the inferior frontal gyrus might be associated with an involuntary amplification or contrast enhancement mechanism, regulating the auditory change detection system. Bayard et al. reported that $A D H D$ symptoms were negatively correlated with gray matter volume in the bilateral inferior frontal cortex [83]. In terms of the orbitofrontal region, including the orbital gyrus, cortical thinning and volumetric reduction of this region have been reported in adult patients with ADHD [74, 84]. In addition, the orbitofrontal region was revealed to be primarily related to emotional instability and impulsivity in ADHD [84, 85].

Furthermore, the insula is known to play a generic role in updating information and cognitive control, including attention allocation to behaviorally relevant salient stimuli [86-88]. The functions mediated by the insula are consistently impaired in ADHD, such as cognitive control, sustained attention, and saliency detection $[89,90]$. One previous volumetric study revealed that youths with ADHD showed a bilateral reduction in the insula region associated with attention problems and inhibition [91]. The superior temporal region has been reported to be abnormal in ADHD. Imaging studies using oddball involuntary attention tasks showed reduced activation in the bilateral superior temporal gyrus in adolescents with ADHD [92, 93]. Previous studies have demonstrated that there are delays in cortical maturation in the bilaterally superior temporal gyrus of ADHD patients that could affect the control of attention [94, 95].

These abnormal regions belonging to frontal, temporal, and limbic lobes are highly related to the pathophysiology of ADHD.
The previous findings support our results regarding altered source activation for MMN in adult ADHD patients and correlations between source activation and ADHD symptom scores. Decreased MMN source activation in adult patients with ADHD could be interpreted as decreased efficiency of automatically regulated attentional processes and increased demand for more effort and resources.

We applied machine learning methods to classify adult ADHD patients and HCs using sensor- and source-level features extracted from MMN ERP data. The best classification performance was achieved when source-level features were used (accuracy: $81.01 \%$, sensitivity: $82.35 \%$, sensitivity: $80.00 \%$ ). According to our results, the source-level features from MMN might play a more important role in the classification of ADHD patients and HCs than the sensor-level features. This might be due to the notion that the shortcoming of sensor-level features could be supplemented by source-level features such as low spatial resolution originating from volume conduction and poor signal-to-noise ratio [96-98]. Thus, the improved spatial information from the source-imaging method might contribute to the enhanced classification performance. When the best classification performance was achieved in the classification of the patients and $\mathrm{HCs}$ using source-level features, 14 regions were selected (bilateral anterior cingulate cortex, bilateral inferior frontal gyrus, bilateral rectal gyrus, bilateral subcallosal gyrus, bilateral extra nuclear, bilateral orbital gyrus, right uncus, and right superior temporal gyrus). MMN source activation in the frontal, temporal, and limbic lobes was dysfunctional in adult ADHD patients. Our results suggest that the reduced MMN activations in these regions could be utilized as important neuromarkers for classifying adult ADHD patients from $\mathrm{HCs}$ and could help to elucidate the neural mechanism of adult ADHD during pre-attentive processing.

Moreover, when we used both sensor- and source-level features for the classification of adult ADHD patients and $\mathrm{HCs}$, we obtained a classification accuracy of $81.01 \%$, which was the same value as that from source-level features. However, the sensitivity was higher for source-level features $(82.35 \%)$ than for both sensor- and source-level features (79.41\%). In the clinical setting, the value of sensitivity, referring to the ability to correctly identify patients with the disease, could be considered more important [99]. Therefore, although the accuracies from source-level features and both sensor- and source-level features were the same, the source-level features might play a more crucial role in the clinical setting as biomarkers for adult ADHD patients.

In this study, a number of MMN features including sensor and source levels showed significant between-group differences based on the univariate analysis method $(p<0.001)$. The statistics for the univariate analyses were calculated based on the differences of mean and standard deviation between two groups. Thus, even though the data distribution of the two groups from the features was overlapped, the features could show significant betweengroup differences with their mean and standard deviation differences. However, to achieve higher classification performances using machine learning, the distribution of data between two groups should be less overlapped. We achieved the classification accuracy of $81.01 \%$ between adult ADHD patients and HCs using the source-level feature set. This classification accuracy was acceptable and we found that the accuracy was significantly based on the permutation test. Since our study was the first to investigate MMN changes in adult patients with ADHD, further studies with a larger number of adult patients with ADHD are warranted to verify our findings.

This study has several limitations. First, when interpreting the results, the relatively small sample size should be considered. Further studies are warranted to validate the findings with larger samples. Second, the patient group and HCs were not matched for education years. However, the difference in education years between the groups has been accepted in recent studies [100], and we controlled 
the variable as a covariate in our statistical analyses. Third, we did not implement individual head models for EEG source imaging. Despite the above limitations, this study is noteworthy as the first attempt to investigate the differences in MMN activities in adult patients with ADHD and HCs. Altered MMN features at both the sensor and source levels were found in ADHD patients compared to HCs, and significant correlations between MMN source activities and ADHD symptom scores were observed. Furthermore, we achieved acceptable classification performances using MMN features for discriminating between the groups. Our results showed the possibility of MMN features as biomarkers for adult ADHD patients, suggesting that the MMN might offer additional useful information for detecting patients in the clinical setting. As a future study, we will attempt to improve classification performances based on deep-learning techniques with larger sample sizes.

\section{REFERENCES}

1. Feldman HM, Reiff MI. Attention deficit-hyperactivity disorder in children and adolescents. N. Engl J Med. 2014;370:838-46.

2. Faraone SV, Biederman J, Mick E. The age-dependent decline of attention deficit hyperactivity disorder: a meta-analysis of follow-up studies. Psychological Med. 2006;36:159-65.

3. Biederman J, Faraone S. Attention-deficit hyperactivity disorder. Lancet. 2005;366:237-48

4. Bush G. Attention-deficit/hyperactivity disorder and attention networks. Neuropsychopharmacology. 2010;35:278-300.

5. McIntosh D, Kutcher S, Binder C, Levitt A, Fallu A, Rosenbluth M. Adult ADHD and comorbid depression: a consensus-derived diagnostic algorithm for ADHD. Neuropsychiatr Dis Treat. 2009;5:137-50.

6. Kiiski H, Rueda-Delgado LM, Bennett M, Knight R, Rai L, Roddy D, et al. Functional EEG connectivity is a neuromarker for adult attention deficit hyperactivity disorder symptoms. Clin Neurophysiol. 2020;131:330-42.

7. Cheng $\mathrm{C}-\mathrm{H}$, Chan P-YS, Hsieh Y-W, Chen K-F. A meta-analysis of mismatch negativity in children with attention deficit-hyperactivity disorders. Neurosci Lett. 2016;612:132-7.

8. Näätänen R, Gaillard AW, Mäntysalo S. Early selective-attention effect on evoked potential reinterpreted. Acta Psychologica. 1978;42:313-29.

9. Javitt DC, Zukin SR, Heresco-Levy U, Umbricht D. Has an angel shown the way? Etiological and therapeutic implications of the PCP/NMDA model of schizophrenia. Schizophrenia Bull. 2012;38:958-66.

10. Näätänen R, Kujala T, Escera $C$, Baldeweg T, Kreegipuu $K$, Carlson $S$, et al. The mismatch negativity (MMN)-a unique window to disturbed central auditory processing in ageing and different clinical conditions. Clin Neurophysiol. 2012;123:424-58

11. Chitty KM, Lagopoulos J, Lee RS, Hickie IB, Hermens DF. A systematic review and meta-analysis of proton magnetic resonance spectroscopy and mismatch negativity in bipolar disorder. Eur Neuropsychopharmacol. 2013;23:1348-63.

12. Javitt DC, Doneshka P, Grochowski S, Ritter W. Impaired mismatch negativity generation reflects widespread dysfunction of working memory in schizophrenia. Arch Gen Psychiatry. 1995;52:550-8.

13. Lakhan SE, Caro M, Hadzimichalis N. NMDA receptor activity in neuropsychiatric disorders. Front Psychiatry. 2013;4:52.

14. Peyrovian B, Rosenblat JD, Pan Z, lacobucci M, Brietzke E, Mclntyre RS. The glycine site of NMDA receptors: a target for cognitive enhancement in psychiatric disorders. Prog Neuro-Psychopharmacol Biol Psychiatry. 2019;92:387-404.

15. Pei-Chen Chang J, Lane H-Y, Tsai E. G. Attention deficit hyperactivity disorder and N-methyl-D-aspartate (NMDA) dysregulation. Curr Pharm Des. 2014;20:5180-5.

16. Winsberg BG, Javitt DC, Shanahan G. Electrophysiological indices of information processing in methylphenidate responders. Biol Psychiatry. 1997;42:434-45.

17. Kilpeläinen R, Partanen J, Karhu J. Reduced mismatch negativity (MMN) suggests deficits in preattentive auditory processing in distractible children. Neuroreport. 1999;10:3341-5.

18. Rothenberger A, Banaschewski T, Heinrich $H$, Moll GH, Schmidt MH, van't Klooster B. Comorbidity in ADHD-children: effects of coexisting conduct disorder or tic disorder on event-related brain potentials in an auditory selectiveattention task. Eur Arch Psychiatry Clin Neurosci. 2000;250:101-10.

19. Huttunen-Scott T, Kaartinen J, Tolvanen A, Lyytinen H. Mismatch negativity (MMN) elicited by duration deviations in children with reading disorder, attention deficit or both. Int J Psychophysiol. 2008;69:69-77.
20. Gomes H, Duff M, Flores A, Halperin JM. Automatic processing of duration in children with attention-deficit/hyperactivity disorder. J Int Neuropsychological Soc: JINS. 2013;19:686-94.

21. Markovska-Simoska S, Pop-Jordanova N. Quantitative EEG in children and adults with attention deficit hyperactivity disorder: comparison of absolute and relative power spectra and theta/beta ratio. Clin EEG Neurosci. 2017;48:20-32.

22. Gentile JP, Atiq R, Gillig PM. Adult ADHD: diagnosis, differential diagnosis, and medication management. Psychiatry. 2006;3:25-30.

23. Mueller A, Candrian G, Kropotov JD, Ponomarev VA, Baschera GM. Classification of $A D H D$ patients on the basis of independent ERP components using a machine learning system. Nonlinear Biomed Phys. BioMed Central. 2010;4:1-12.

24. Mueller A, Candrian G, Grane VA, Kropotov JD, Ponomarev VA, Baschera GM. Discriminating between ADHD adults and controls using independent ERP components and a support vector machine: a validation study. Nonlinear Biomed Phys. 2011;5:5.

25. Tenev A, Markovska-Simoska S, Kocarev L, Pop-Jordanov J, Müller A, Candrian G. Machine learning approach for classification of ADHD adults. Int J Psychophysiol. 2014;93:162-6.

26. Poil S-S, Bollmann S, Ghisleni C, O'Gorman RL, Klaver P, Ball J, et al. Age dependent electroencephalographic changes in attention-deficit/hyperactivity disorder (ADHD). Clin Neurophysiol. 2014;125:1626-38.

27. Burkey JM, Lippy WH, Schuring AG, Rizer FM. Clinical utility of the $512-\mathrm{Hz}$ Rinne tuning fork test. Am J Otol. 1998;19:59-62.

28. Heo S, Kim JH, Joung YS, Lee WI, Kim JJ, Sohn SH, et al. Clinical utility of the Korean version of the WHO adult attention-deficit/hyperactivity disorder selfreport scale screener. Psychiatry Investig. 2018;15:325-9.

29. Kessler RC, Adler L, Ames M, Demler O, Faraone S, Hiripi E, et al. The World Health Organization Adult ADHD Self-Report Scale (ASRS): a short screening scale for use in the general population. Psychological Med. 2005;35:245-56.

30. Semlitsch HV, Anderer P, Schuster P, Presslich O. A solution for reliable and valid reduction of ocular artifacts, applied to the P300 ERP. Psychophysiology. 1986;23:695-703.

31. Light GA, Braff DL. Mismatch negativity deficits are associated with poor functioning in schizophrenia patients. Arch Gen Psychiatry. 2005;62:127-36.

32. Şevik $A E$, Anıl Yağcıoğlu AE, Yağcıoğlu S, Karahan S, Gürses N, Yıldız M. Neuropsychological performance and auditory event related potentials in schizophrenia patients and their siblings: a family study. Schizophrenia Res. 2011;130:195-202.

33. Lee S-H, Sung K, Lee K-S, Moon E, Kim C-G. Mismatch negativity is a stronger indicator of functional outcomes than neurocognition or theory of mind in patients with schizophrenia. Prog Neuro-Psychopharmacol Biol Psychiatry. 2014;48:213-9.

34. Pascual-Marqui RD. Standardized low-resolution brain electromagnetic tomography (sLORETA): technical details. Methods Find Exp Clin Pharm. 2002;24:5-12.

35. Fuchs $M$, Kastner J, Wagner M, Hawes $S$, Ebersole JS. A standardized boundary element method volume conductor model. Clin Neurophysiol. 2002;113:702-12.

36. Brett M, Johnsrude IS, Owen AM. The problem of functional localization in the human brain. Nat Rev Neurosci. 2002;3:243-9.

37. Deouell LY, Bentin S, Giard MH. Mismatch negativity in dichotic listening: evidence for interhemispheric differences and multiple generators. Psychophysiology. 1998;35:355-65.

38. Rinne T, Alho K, Ilmoniemi R, Virtanen J, Näätänen R. Separate time behaviors of the temporal and frontal mismatch negativity sources. Neuroimage. 2000;12:14-19.

39. Jemel B, Achenbach C, Müller BW, Röpcke B, Oades RD. Mismatch negativity results from bilateral asymmetric dipole sources in the frontal and temporal lobes. Brain Topogr. 2002;15:13-27.

40. Park HJ, Kwon JS, Youn T, Pae JS, Kim JJ, Kim MS, et al. Statistical parametric mapping of LORETA using high density EEG and individual MRI: application to mismatch negativities in schizophrenia. Hum Brain Mapp. 2002;17:168-78.

41. Doeller CF, Opitz B, Mecklinger A, Krick C, Reith W, Schröger E. Prefrontal cortex involvement in preattentive auditory deviance detection:: neuroimaging and electrophysiological evidence. Neuroimage. 2003;20:1270-82.

42. Oknina LB, Wild-Wall N, Oades RD, Juran SA, Röpcke B, Pfueller U, et al. Frontal and temporal sources of mismatch negativity in healthy controls, patients at onset of schizophrenia in adolescence and others at 15 years after onset. Schizophrenia Res. 2005;76:25-41.

43. Oades RD, Wild-Wall N, Juran SA, Sachsse J, Oknina LB, Röpcke B. Auditory change detection in schizophrenia: sources of activity, related neuropsychological function and symptoms in patients with a first episode in adolescence, and patients 14 years after an adolescent illness-onset. BMC Psychiatry. 2006;6:7.

44. Schönwiesner M, Novitski N, Pakarinen S, Carlson S, Tervaniemi M, Näätänen R. Heschl's gyrus, posterior superior temporal gyrus, and mid-ventrolateral 
prefrontal cortex have different roles in the detection of acoustic changes. J Neurophysiol. 2007;97:2075-82.

45. Schnyer DM, Clasen PC, Gonzalez C, Beevers CG. Evaluating the diagnostic utility of applying a machine learning algorithm to diffusion tensor MRI measures in individuals with major depressive disorder. Psychiatry Res: Neuroimaging. 2017;264:1-9.

46. Kim H, Kim L, Im C-H. Machine-learning-based detection of craving for gaming using multimodal physiological signals: Validation of test-retest reliability for practical use. Sensors. 2019;19:3475.

47. Chen Q-F, Zou T-X, Yang Z-T, Chen H-J. Identification of patients with and without minimal hepatic encephalopathy based on gray matter volumetry using a support vector machine learning algorithm. Sci Rep. 2020;10:1-8.

48. Guo Y, Qiu J, Lu W. Support vector machine-based schizophrenia classification using morphological information from amygdaloid and hippocampal subregions. Brain Sci. 2020;10:562.

49. Steardo L Jr, Carbone EA, de Filippis R, Pisanu C, Segura-Garcia C, Squassina A, et al. Application of support vector machine on fMRI data as biomarkers in schizophrenia diagnosis: a systematic review. Front Psychiatry. 2020;11:588.

50. Bracher-Smith $M$, Crawford $K$, Escott-Price V. Machine learning for genetic prediction of psychiatric disorders: a systematic review. Mol Psychiatry. 2021;26:70-79.

51. Wottschel V, Chard DT, Enzinger C, Filippi M, Frederiksen JL, Gasperini C, et al. SVM recursive feature elimination analyses of structural brain MRI predicts nearterm relapses in patients with clinically isolated syndromes suggestive of multiple sclerosis. Neurolmage: Clin. 2019;24:102011.

52. Tong F, Shahid M, Jin P, Jung S, Kim WH, Kim J, et al. Classification of the urinary metabolome using machine learning and potential applications to diagnosing interstitial cystitis. Bladder. 2020;7:e43.

53. Zhang J, Li Y, Gao Y, Hu J, Huang B, Rong S, et al. An SBM-based machine learning model for identifying mild cognitive impairment in patients with Parkinson's disease. J Neurological Sci. 2020;418:117077.

54. Gu Q, Li Z, Han J. Generalized fisher score for feature selection. arXiv: 1202.3725 [Preprint]. 2012. Available from https://arxiv.org/abs/1202.3725.

55. Alimardani F, Cho J-H, Boostani R, Hwang H-J. Classification of bipolar disorder and schizophrenia using steady-state visual evoked potential based features. IEEE Access. 2018;6:40379-88.

56. Shim $\mathrm{M}$, Jin MJ, Im C-H, Lee $\mathrm{S}-\mathrm{H}$. Machine-learning-based classification between post-traumatic stress disorder and major depressive disorder using P300 features. Neurolmage: Clin. 2019;24:102001.

57. Xu Z, Yang X, Gao M, Liu L, Sun J, Liu P, et al. Abnormal resting-state functional connectivity in the whole brain in lifelong premature ejaculation patients based on machine learning approach. Front Neurosci. 2019;13:448.

58. Tong Y, Huang X, Qi C-X, Shen Y. Altered functional connectivity of the primary visual cortex in patients with iridocyclitis and assessment of its predictive value using machine learning. Front Immunol. 2021;12:1650.

59. Yamamuro K, Ota T, lida J, Nakanishi Y, Kishimoto N, Kishimoto T. Associations between the mismatch-negativity component and symptom severity in children and adolescents with attention deficit/hyperactivity disorder. Neuropsychiatr Dis Treat. 2016;12:3183-90.

60. Näätänen R. The mismatch negativity: a powerful tool for cognitive neuroscience. Ear Hearing. 1995;16:6-18.

61. Rydkjær J, Møllegaard Jepsen JR, Pagsberg AK, Fagerlund B, Glenthøj BY, Oranje B. Mismatch negativity and P3a amplitude in young adolescents with firstepisode psychosis: a comparison with ADHD. Psychological Med. 2017;47:377-88.

62. Levänen S, Ahonen A, Hari R, McEvoy L, Sams M. Deviant auditory stimuli activate human left and right auditory cortex differently. Cereb Cortex. 1996;6:288-96

63. Alho K, Woods DL, Algazi A, Knight R, Näätänen R. Lesions of frontal cortex diminish the auditory mismatch negativity. Electroencephalogr Clin Neurophysiol. 1994;91:353-62.

64. Waberski TD, Kreitschmann-Andermahr I, Kawohl W, Darvas F, Ryang Y, Rodewald $M$, et al. Spatio-temporal source imaging reveals subcomponents of the human auditory mismatch negativity in the cingulum and right inferior temporal gyrus. Neurosci Lett. 2001;308:107-10.

65. Garrido MI, Kilner JM, Stephan KE, Friston KJ. The mismatch negativity: a review of underlying mechanisms. Clin Neurophysiol. 2009;120:453-63.

66. Paavilainen $P$, Mikkonen $M$, Kilpeläinen $M$, Lehtinen $R$, Saarela $M$, Tapola $L$. Evidence for the different additivity of the temporal and frontal generators of mismatch negativity: a human auditory event-related potential study. Neurosci Lett. 2003;349:79-82

67. Giard $\mathrm{MH}$, Perrin F, Pernier J, Bouchet P. Brain generators implicated in the processing of auditory stimulus deviance: a topographic event-related potential study. Psychophysiology. 1990;27:627-40.
68. Gomot $M$, Giard $M-H$, Roux S, Barthélémy $C$, Bruneau N. Maturation of frontal and temporal components of mismatch negativity (MMN) in children. Neuroreport. 2000;11:3109-12.

69. Maess B, Jacobsen T, Schröger E, Friederici AD. Localizing pre-attentive auditory memory-based comparison: magnetic mismatch negativity to pitch change. Neuroimage. 2007;37:561-71.

70. Rubia K, Overmeyer S, Taylor E, Brammer M, Williams SC, Simmons A, et al. Hypofrontality in attention deficit hyperactivity disorder during higher-order motor control: a study with functional MRI. Am J Psychiatry. 1999;156:891-6.

71. Kofler MJ, Rapport MD, Bolden J, Sarver DE, Raiker JS. ADHD and working memory: the impact of central executive deficits and exceeding storage/ rehearsal capacity on observed inattentive behavior. J Abnorm child Psychol. 2010;38:149-61.

72. Badgaiyan RD, Sinha S, Sajjad M, Wack DS. Attenuated tonic and enhanced phasic release of dopamine in attention deficit hyperactivity disorder. PLoS One. 2015; 10:e0137326.

73. Seidman $\amalg$, Valera EM, Makris N. Structural brain imaging of attention-deficit/ hyperactivity disorder. Biol Psychiatry. 2005;57:1263-72.

74. Makris N, Biederman J, Valera EM, Bush G, Kaiser J, Kennedy DN, et al. Cortical thinning of the attention and executive function networks in adults with attention-deficit/hyperactivity disorder. Cereb Cortex. 2006;17:1364-75.

75. Seidman LJ, Valera EM, Makris N, Monuteaux MC, Boriel DL, Kelkar K, et al. Dorsolateral prefrontal and anterior cingulate cortex volumetric abnormalities in adults with attention-deficit/hyperactivity disorder identified by magnetic resonance imaging. Biol Psychiatry. 2006;60:1071-80.

76. Amico F, Stauber J, Koutsouleris N, Frodl T. Anterior cingulate cortex gray matter abnormalities in adults with attention deficit hyperactivity disorder: a voxel-based morphometry study. Psychiatry Res: Neuroimaging. 2011;191:31-35.

77. Seidman L, Biederman J, Liang L, Valera EM, Monuteaux MC, Brown A, et al. Gray matter alterations in adults with attention-deficit/hyperactivity disorder identified by voxel based morphometry. Biol Psychiatry. 2011;69:857-66.

78. Bush G, Frazier JA, Rauch SL, Seidman LJ, Whalen PJ, Jenike MA, et al. Anterior cingulate cortex dysfunction in attention-deficit/hyperactivity disorder revealed by fMRI and the Counting Stroop. Biol Psychiatry. 1999;45:1542-52.

79. Makris N, Seidman L, Valera EM, Biederman J, Monuteaux MC, Kennedy DN, et al. Anterior cingulate volumetric alterations in treatment-naive adults with ADHD: a pilot study. J Atten Disord. 2010;13:407-13.

80. Aron AR, Fletcher PC, Bullmore ET, Sahakian BJ, Robbins TW. Stop-signal inhibition disrupted by damage to right inferior frontal gyrus in humans. Nat Neurosci. 2003;6:115-6.

81. Aron AR, Poldrack RA. Cortical and subcortical contributions to stop signal response inhibition: role of the subthalamic nucleus. J Neurosci. 2006;26:2424-33.

82. Opitz B, Rinne T, Mecklinger A, Von Cramon DY, Schröger E. Differential contribution of frontal and temporal cortices to auditory change detection: fMRI and ERP results. Neuroimage. 2002;15:167-74.

83. Bayard F, Thunell CN, Abé C, Almeida R, Banaschewski T, Barker G, et al. Distinct brain structure and behavior related to $A D H D$ and conduct disorder traits. Mol Psychiatry. 2020;25:3020-33.

84. Hesslinger B, Tebartz van Elst L, Thiel T, Haegele K, Hennig J, Ebert D. Frontoorbital volume reductions in adult patients with attention deficit hyperactivity disorder. Neurosci Lett. 2002;328:319-21.

85. Barkley RA. Behavioral inhibition, sustained attention, and executive functions: constructing a unifying theory of ADHD. Psychological Bull. 1997; 121:65-94.

86. Derrfuss J, Brass M, Neumann J, von Cramon DY. Involvement of the inferior frontal junction in cognitive control: Meta-analyses of switching and Stroop studies. Hum Brain Mapp. 2005;25:22-34.

87. Seeley WW, Menon V, Schatzberg AF, Keller J, Glover GH, Kenna H, et al. Dissociable intrinsic connectivity networks for salience processing and executive control. J Neurosci. 2007:27:2349-56.

88. Hampshire A, Chamberlain SR, Monti MM, Duncan J, Owen AM. The role of the right inferior frontal gyrus: inhibition and attentional control. Neuroimage. 2010;50:1313-9.

89. Willcutt EG, Doyle AE, Nigg JT, Faraone SV, Pennington BF. Validity of the executive function theory of attention-deficit/hyperactivity disorder: a metaanalytic review. Biol Psychiatry. 2005;57:1336-46.

90. Rubia K, Alegria AA, Cubillo Al, Smith AB, Brammer MJ, Radua J. Effects of stimulants on brain function in attention-deficit/hyperactivity disorder: a systematic review and meta-analysis. Biol Psychiatry. 2014;76:616-28.

91. Lopez-Larson MP, King JB, Terry J, McGlade EC, Yurgelun-Todd D. Reduced insular volume in attention deficit hyperactivity disorder. Psychiatry Res: Neuroimaging. 2012;204:32-39. 
92. Silk T, Vance A, Rinehart N, Egan G, O'Boyle M, Bradshaw JL, et al. Fronto-parietal activation in attention-deficit hyperactivity disorder, combined type: functional magnetic resonance imaging study. Br J Psychiatry. 2005;187:282-3.

93. Rubia K, Smith AB, Brammer MJ, Taylor E. Temporal lobe dysfunction in medication-naive boys with attention-deficit/hyperactivity disorder during attention allocation and its relation to response variability. Biol Psychiatry. 2007;62:999-1006.

94. Shaw P, Eckstrand K, Sharp W, Blumenthal J, Lerch JP, Greenstein D, et al. Attention-deficit/hyperactivity disorder is characterized by a delay in cortical maturation. Proc Natl Acad Sci USA. 2007;104:19649-54.

95. Lei D, Ma J, Du X, Shen G, Jin X, Gong Q. Microstructural abnormalities in the combined and inattentive subtypes of attention deficit hyperactivity disorder: a diffusion tensor imaging study. Sci Rep. 2014;4:6875.

96. van den Broek SP, Reinders F, Donderwinkel M, Peters M. Volume conduction effects in EEG and MEG. Electroencephalogr Clin Neurophysiol. 1998;106: 522-34.

97. Nolte G, Bai O, Wheaton L, Mari Z, Vorbach S, Hallett M. Identifying true brain interaction from EEG data using the imaginary part of coherency. Clin Neurophysiol. 2004;115:2292-307.

98. Lemm S, Curio G, Hlushchuk Y, Muller K-R. Enhancing the signal-to-noise ratio of ICA-based extracted ERPs. IEEE Trans Biomed Eng. 2006;53:601-7.

99. Lalkhen AG, McCluskey A. Clinical tests: sensitivity and specificity. Continuing Educ Anaesth Crit Care Pain. 2008;8:221-3.

100. Kim S, Kim JS, Kwon YJ, Lee HY, Yoo JH, Lee YJ, et al. Altered cortical functional network in drug-naive adult male patients with attention-deficit hyperactivity disorder: a resting-state electroencephalographic study. Prog NeuroPsychopharmacol Biol Psychiatry. 2020;110056:110056.

\section{ACKNOWLEDGEMENTS}

This work was supported by a grant (2020R111A3068017) awarded by the Basic Science Research Program through the National Research Foundation of Korea (NRF) and funded by the Ministry of Education. This study was also supported by Soonchunhyang University.

\section{AUTHOR CONTRIBUTIONS}

SK analyzed the data and wrote the paper. JSK designed the study and wrote the paper. SHS, YJK, and HYL collected the data. JSK, JHB, and JHY reviewed and revised the paper.

\section{COMPETING INTERESTS}

The authors declare no competing interests.

\section{ADDITIONAL INFORMATION}

Correspondence and requests for materials should be addressed to Se-hoon Shim or Ji Sun Kim.

Reprints and permission information is available at http://www.nature.com/ reprints

Publisher's note Springer Nature remains neutral with regard to jurisdictional claims in published maps and institutional affiliations.

(i) Open Access This article is licensed under a Creative Commons adaptation, distribution and reproduction in any medium or format, as long as you give appropriate credit to the original author(s) and the source, provide a link to the Creative Commons license, and indicate if changes were made. The images or other third party material in this article are included in the article's Creative Commons license, unless indicated otherwise in a credit line to the material. If material is not included in the article's Creative Commons license and your intended use is not permitted by statutory regulation or exceeds the permitted use, you will need to obtain permission directly from the copyright holder. To view a copy of this license, visit http://creativecommons. org/licenses/by/4.0/.

(c) The Author(s) 2021 